\title{
Possible Proton Conduction Mechanism in Pseudo-Protic Ionic Liquids: The Concept of Specific Proton Conduction
}

\author{
Hikari Watanabe, ${ }^{\dagger}$ Tatsuya Umecky, ${ }^{\ddagger}$ Nana Arai,${ }^{\dagger}$ Andreas Nazet, ${ }^{\S}$ Thomas \\ Sonnleitner, ${ }^{\S}$ Toshiyuki Takamuku, ${ }^{\ddagger}$ Kenneth R Harris, ${ }^{\natural}$ Yasuo Kameda, ${ }^{\perp}$ Richard \\ Buchner*§ and Yasuhiro Umebayashi* ${ }^{\dagger}$
}

†raduate School of Science and Technology, Niigata University, 8050 Ikarashi, 2-no-cho, Nishi-ku, Niigata City, 950-2181, Japan

${ }^{\ddagger}$ Graduate School of Science and Engineering, Saga University, 1 Honjo-machi, Saga, 840-8502, Japan

${ }^{\S}$ Institute of Theoretical and Physical Chemistry, University of Regensburg, Universitätsstr. 31, 93053 Regensburg, Germany

"School of Science, The University of New South Wales, PO Box 7916, Canberra BC, Australian Capital Territory 2610, Australia

${ }^{\perp}$ Department of Material and Biological Chemistry, Faculty of Science, Yamagata University, 1-4-12, Kojirakawa-machi, Yamagata City, Yamagata 990-8560, Japan 
Table S1 Experimental Transport Coefficients.

\begin{tabular}{cccccccc}
\hline$T / \mathrm{K}$ & $10^{12} \mathrm{DS}+$ & $10^{12} \mathrm{Ds}-$ & $\rho^{a}$ & $c$ & $\eta^{b}$ & $\kappa$ & $\Lambda^{c}$ \\
& $/ \mathrm{m}^{2} \cdot \mathrm{s}^{-1}$ & $/ \mathrm{m}^{2} \cdot \mathrm{s}^{-1}$ & $/ \mathrm{kg} \cdot \mathrm{m}^{-3}$ & $/ \mathrm{mol} \cdot \mathrm{L}^{-1}$ & $/ \mathrm{mPa} \cdot \mathrm{s}$ & $/ \mathrm{S} \cdot \mathrm{m}^{-1}$ & $/ \mu \mathrm{S} \cdot \mathrm{m}^{2} \cdot \mathrm{mol}^{-1}$ \\
\hline 268.15 & & & 1100.1 & 7.738 & 22.15 & 0.208 & 26.9 \\
273.15 & & 1095.1 & 7.703 & 17.59 & 0.254 & 33.0 \\
278.15 & & 1090.2 & 7.669 & 14.21 & 0.305 & 39.8 \\
283.15 & & 1085.5 & 7.636 & 11.64 & 0.362 & 47.4 \\
288.15 & & 1080.9 & 7.604 & 9.67 & 0.425 & 55.8 \\
293.15 & 233 & 197 & 1076.4 & 7.572 & 8.14 & 0.492 & 65.0 \\
298.15 & 369 & 324 & 1072.0 & 7.541 & 6.92 & 0.565 & 75.0 \\
313.15 & 454 & 400 & 1059.6 & 7.454 & 4.52 & 0.812 & 109 \\
323.15 & 584 & 509 & 1051.9 & 7.400 & 3.55 & 0.997 & 135 \\
333.15 & & & 1044.7 & 7.349 & 2.87 & 1.19 & 163 \\
343.16 & & & & & & & \\
$100 \cdot u_{\mathrm{r}}{ }^{2}$ & 2 & 2 & 0.03 & 0.03 & 3.4 & 5.2 & 192 \\
\hline
\end{tabular}

${ }^{a}$ Calculated from a binomial fit to the data of Doi et al. ${ }^{1}: \rho / \mathrm{g} \cdot \mathrm{cm}^{-3}=1.0951-9.9816 \cdot 10^{-4}(T / \mathrm{K}$ $-273.15)+2.366 \cdot 10^{-6}(T / \mathrm{K}-273.15)^{2}$, st. devn $=0.0003 \mathrm{~g} \cdot \mathrm{cm}^{-3}, 258<T / \mathrm{K}<343{ }^{b}$ Calculated from a Litovitz fit to the data of Doi et al. ${ }^{1}: \ln (\eta / \mathrm{mPa} \cdot \mathrm{s})=(1.171 \pm 0.056)+(82.3 \pm 1.5) \cdot 10^{-6} /(T / \mathrm{K})^{3}$, st. devn $=3.4 \%, 268<T / \mathrm{K}<343 .{ }^{c}$ Calculated from a Litovitz fit to the data of Doi et al. ${ }^{1}: \ln (\Lambda /$ $\left.\mu \mathrm{S} \cdot \mathrm{m}^{2} \cdot \mathrm{mol}^{-1}\right)=(7.050 \pm 0.087)+(72.4 \pm 2.3) 10^{-6} /(T / \mathrm{K})^{3}$, st. devn $=5.2 \%, 268<T / \mathrm{K}<343$. These equations give a better representation of the data than those used by Doi et al. Their points below $268 \mathrm{~K}$ appear to be too high in the case of the viscosity and too low in the case of the conductivity and have not been used in obtaining the equations above. ${ }^{d} \mathrm{u}_{\mathrm{r}}$ represents actual uncertainty, not a percentage. 
Table S2 Experimental Transport Coefficients.Velocity Correlation $\left(f_{\mathrm{ij}}\right)$, and Distinct Diffusion $\left(D^{d}{ }_{++}\right)$Coefficients, Laity Resistance Coefficients and Nernst-Einstein $\Delta$

\begin{tabular}{|c|c|c|c|c|c|c|c|c|c|c|c|}
\hline$T / \mathrm{K}$ & $f_{++}^{a}$ & $f_{--}$ & $f_{+-}$ & $D_{++}^{\mathrm{d}} b$ & $D^{\mathrm{d}}$ & $D^{\mathrm{d}}+$ & $\Delta$ & $r_{++}^{c}$ & $r$ & $r_{+-}$ & $r_{+-}^{2} /\left(r_{++} r_{--}\right)$ \\
\hline 268.15 & & & -0.202 & & & -3.13 & & & & 691 & \\
\hline 273.15 & & & -0.254 & & & -3.91 & & & & 564 & \\
\hline 278.15 & & & -0.313 & & & -4.80 & & & & 468 & \\
\hline 283.15 & & & -0.381 & & & -5.83 & & & & 392 & \\
\hline 288.15 & & & -0.459 & & & -6.98 & & & & 333 & \\
\hline 293.15 & & & -0.546 & & & -8.27 & & & & 286 & \\
\hline 298.15 & -30.41 & -25.22 & -0.643 & -458 & -380 & -9.69 & 0.954 & -227 & -223 & 248 & 1.2 \\
\hline 313.15 & -48.80 & -42.00 & -0.993 & -727 & -626 & -14.8 & 0.956 & -157 & -155 & 171 & 1.2 \\
\hline 323.15 & -60.41 & -52.26 & -1.28 & -894 & -773 & -18.9 & 0.954 & -126 & -125 & 138 & 1.2 \\
\hline 333.15 & -78.30 & -67.01 & -1.60 & -1150 & -985 & -23.5 & 0.956 & -105 & -104 & 115 & 1.2 \\
\hline 343.15 & & & -1.96 & & & -28.6 & & & & 97.0 & \\
\hline $100 \cdot u_{\mathbf{r}}^{d}$ & 3 & 3 & 5 & 3 & 3 & 5 & $0.01^{d}$ & 6 & 6 & 5 & \\
\hline
\end{tabular}

Units: ${ }^{a} f_{\mathrm{ij}}: 10^{-15} \mathrm{~m}^{5} /(\mathrm{mol} \cdot \mathrm{s}) \cdot{ }^{b} \mathrm{D}_{\mathrm{ijj}}: 10^{-12} \mathrm{~m}^{2} / \mathrm{s} .{ }^{c} r_{\mathrm{ij}}: 10^{12} \mathrm{~J} \cdot \mathrm{s} \cdot \mathrm{m}^{-2} \cdot \mathrm{mol}^{-1} \cdot{ }^{d}$ Actual uncertainty, not a percentage.

The velocity cross-correlation coefficients are given by ${ }^{2,3}$

$$
\begin{aligned}
& f_{++} \equiv \frac{N_{\mathrm{A}} V}{3} \int_{0}^{\infty}\left\langle v_{+\alpha}(0) \cdot v_{+\beta}(t)\right\rangle \mathrm{d} t=R T \kappa\left(\frac{M_{-}}{z_{-} F c M}\right)^{2}-\frac{D_{\mathrm{S}+}}{v_{+} c} \\
& f_{--} \equiv \frac{N_{\mathrm{A}} V}{3} \int_{0}^{\infty}\left\langle v_{-\alpha}(0) \cdot v_{-\beta}(t)\right\rangle \mathrm{d} t=R T \kappa\left(\frac{M_{+}}{z_{+} F c M}\right)^{2}-\frac{D_{\mathrm{S}-}}{v_{-} c},
\end{aligned}
$$

And

$$
f_{+-} \equiv \frac{N_{\mathrm{A}} V}{3} \int_{0}^{\infty}\left\langle v_{+\alpha}(0) \cdot v_{-\beta}(t)\right\rangle \mathrm{d} t=R T \kappa \frac{M_{+} M_{-}}{z_{+} z_{-}(F c M)^{2}},
$$

where $N_{\mathrm{A}}$ is the Avogadro constant, $V$ the volume of the ensemble, $\kappa$ is the conductivity, $c$ the

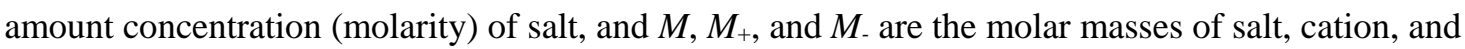
anion, respectively. $v_{i \alpha}$ is the velocity of ion $\alpha$ of species $i,(i=+$ or -$)$, etc.

The distinct diffusion coefficients are given by ${ }^{4}$

$$
D_{i j}^{d}=\left(v_{+}+v_{-}\right) c f_{i j}=v c f_{i j}
$$


Table S3 Dielectric fit parameters for neat $\mathrm{C}_{1} \mathrm{Im}, \mathrm{HOAc}$ and $\left(\mathrm{C}_{1} \mathrm{Im}+\mathrm{AcOH}\right)$ equimolar mixture.

\begin{tabular}{cccccccccccc}
\hline & $\mathrm{T} /{ }^{\circ} \mathrm{C}$ & $\varepsilon_{\mathrm{s}}$ & $S_{1}$ & $\beta_{1}$ & $\tau_{1} / \mathrm{ps}$ & $S_{2}$ & $\alpha 2$ & $\tau_{2} / \mathrm{ps}$ & $S_{3}$ & $\tau_{3} / \mathrm{ps}$ & $\varepsilon_{\infty}$ \\
\hline Neat $\mathrm{C}_{1} \mathrm{Im}$ & 25 & 36.2 & & & & 30.9 & 0.007 & 28.3 & 1.80 & 3.32 & 3.51 \\
Neat $\mathrm{AcOH}$ & 25 & 6.81 & & & & 3.19 & 0.09 & 91.0 & 0.62 & 7.93 & 3.00 \\
$\left(\mathrm{C}_{1} \mathrm{Im}+\mathrm{AcOH}\right)$ & 5 & 31.8 & 24.9 & 0.631 & 882 & 1.84 & & 42.7 & 0.94 & 2.40 & 4.18 \\
& 15 & 32.9 & 24.4 & 0.713 & 594 & 2.97 & & 34.5 & 1.28 & 2.32 & 4.23 \\
& 25 & 31.7 & 23.8 & 0.734 & 416 & 2.50 & & 28.0 & 1.48 & 2.55 & 3.95 \\
& 35 & 30.0 & 20.6 & 0.827 & 273 & 3.65 & & 25.6 & 1.59 & 2.34 & 4.16 \\
& 45 & 27.9 & 18.9 & 0.831 & 230 & 3.21 & & 24.0 & 1.87 & 2.40 & 3.91 \\
& 55 & 28.9 & 18.7 & 0.879 & 171 & 4.44 & & 18.2 & 1.57 & 2.40 & 4.20 \\
& 65 & 27.1 & 17.1 & 0.899 & 130 & 4.21 & & 14.9 & 1.68 & 2.72 & 4.11 \\
\hline
\end{tabular}



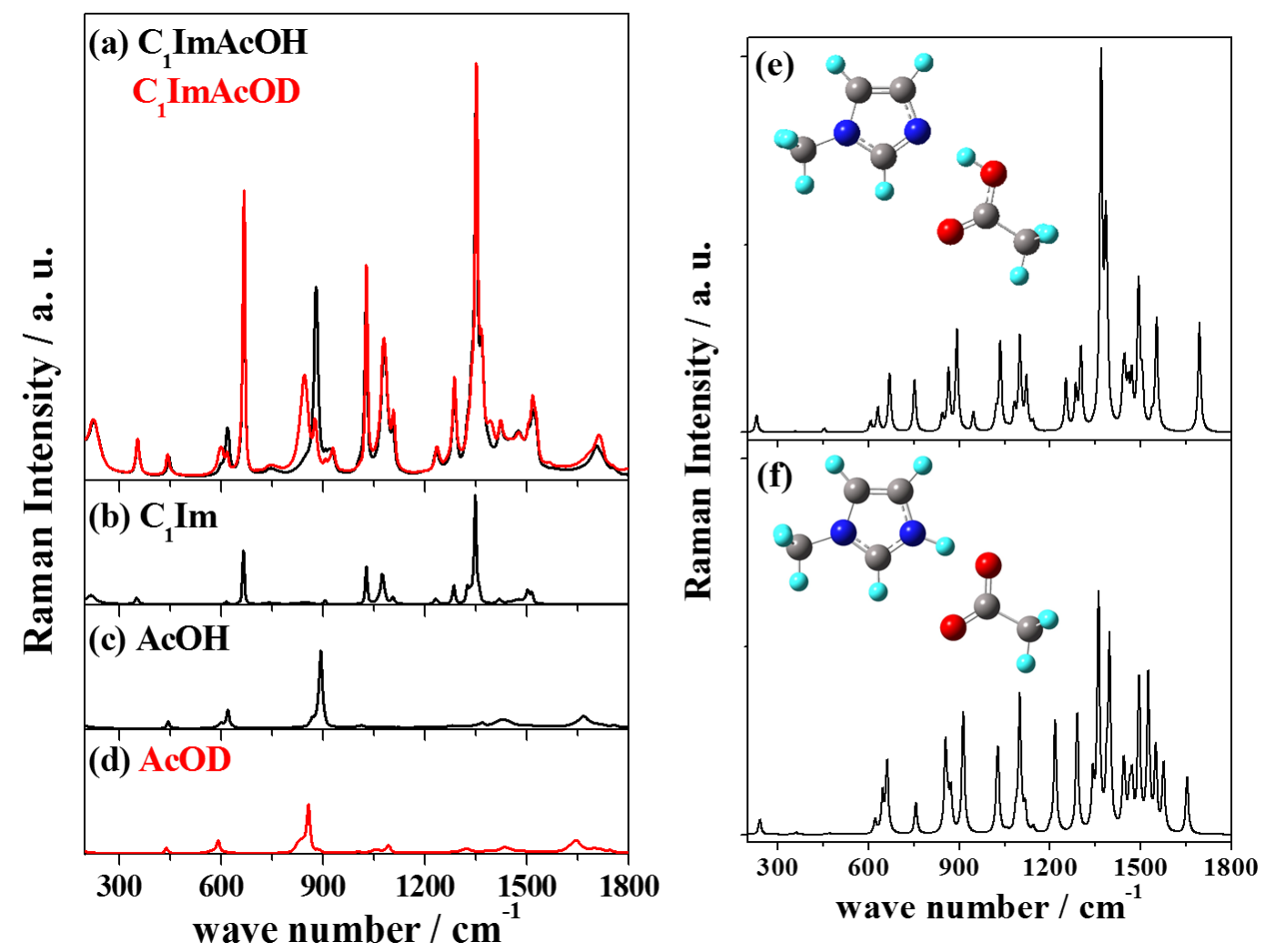

Figure S1 Experimental Raman spectra of (a) $\left(\mathrm{C}_{1} \mathrm{Im}+\mathrm{AcOH}\right)$ equimolar mixture, (b) neat $\mathrm{C}_{1} \mathrm{Im}$, (c) neat $\mathrm{AcOH}$, (d) neat $\mathrm{AcOD}$, respectively. Black and red lines represent the $\left(\mathrm{C}_{1} \mathrm{Im}-\mathrm{AcOH}\right)$ mixture and the corresponding equimolar mixture composed of the deuterated AcOD instead of $\mathrm{AcOH}$. The theoretical Raman spectra of (e) $\left(\mathrm{C}_{1} \mathrm{Im}+\mathrm{AcOH}\right)$ equimolar mixture and (f) $\mathrm{C}_{1} \mathrm{HIm}^{+}$and $\mathrm{AcO}^{-}$ion pair, respectively. 

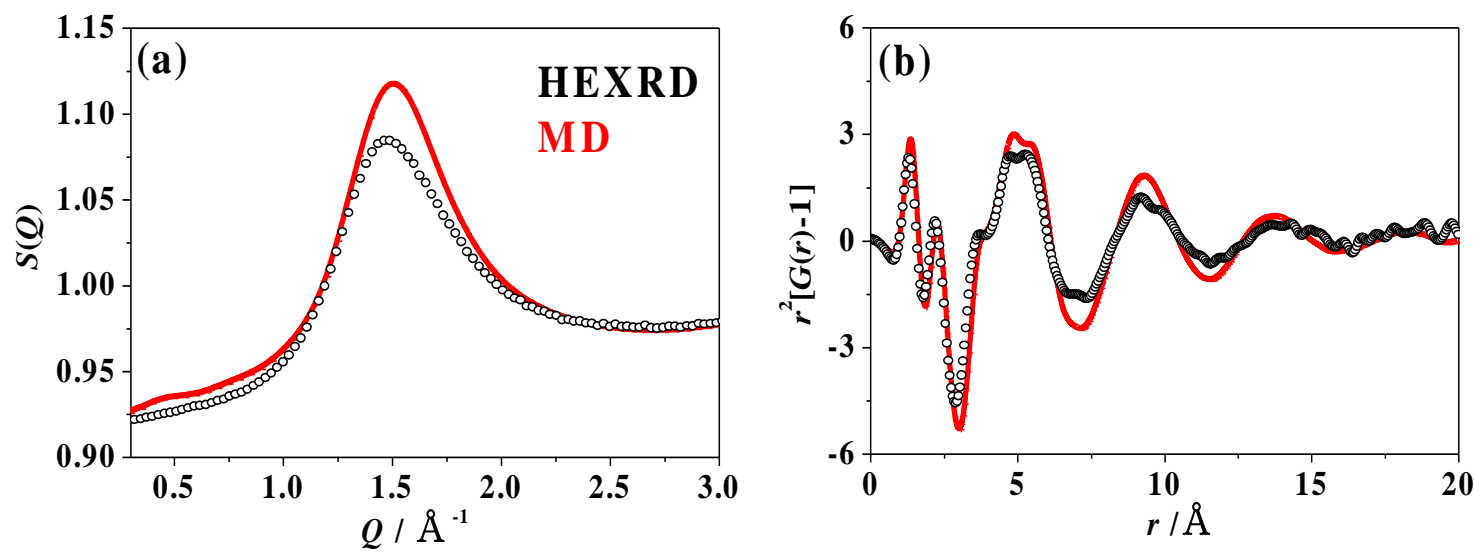

Figure S2 X-ray structure factors at the $Q$ range of $0.3-3.0 \AA^{-1}$ (a) and radial distribution functions as the form of $r^{2}\{G(r)-1\}$ at the $r$ range of $0-20 \AA$ (b) for $\left(\mathrm{C}_{1} \mathrm{Im}+\mathrm{AcOH}\right)$ equimolar mixture at $298 \mathrm{~K}$. Plots and solid lines represent for the experimental and the MD simulated values, respectively. 

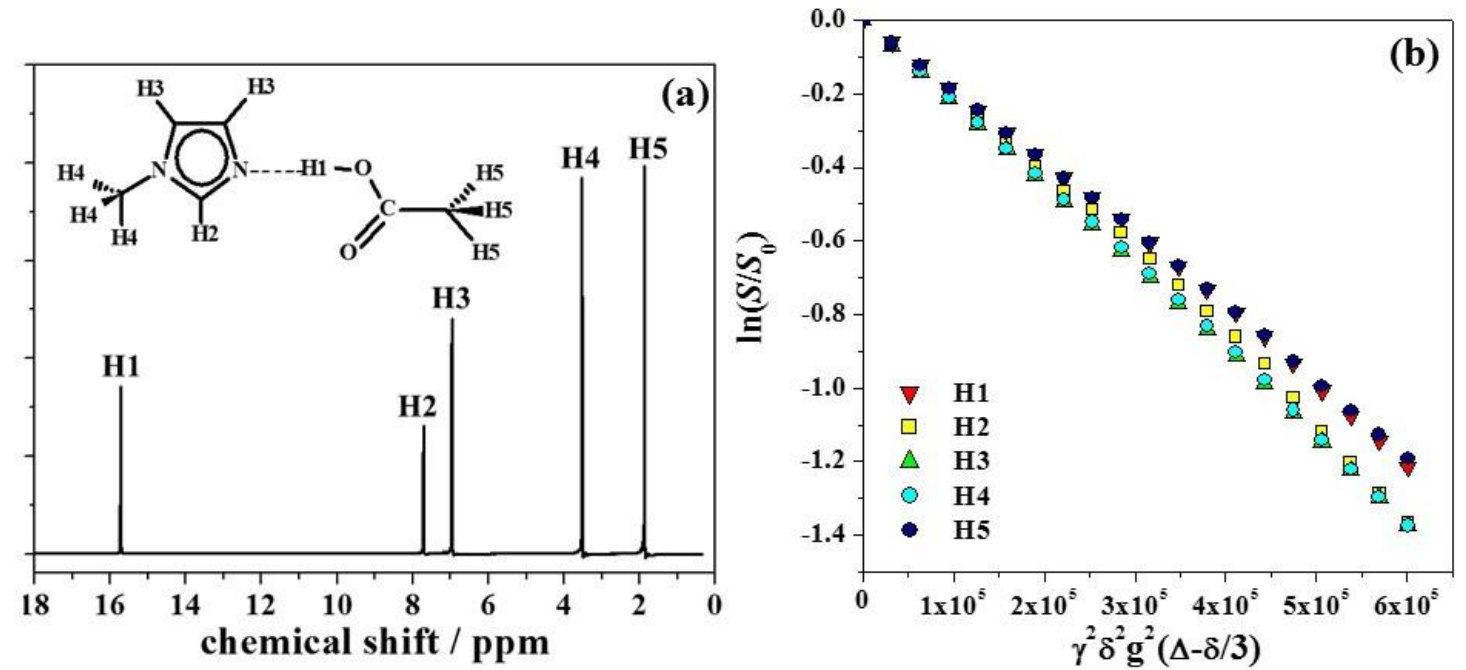

Figure S3 Typical ${ }^{1} \mathrm{H}$ NMR spectrum (a) and the PGSE attenuation plots (b) for $\left(\mathrm{C}_{1} \mathrm{Im}+\mathrm{AcOH}\right)$ at $298 \mathrm{~K}$. 


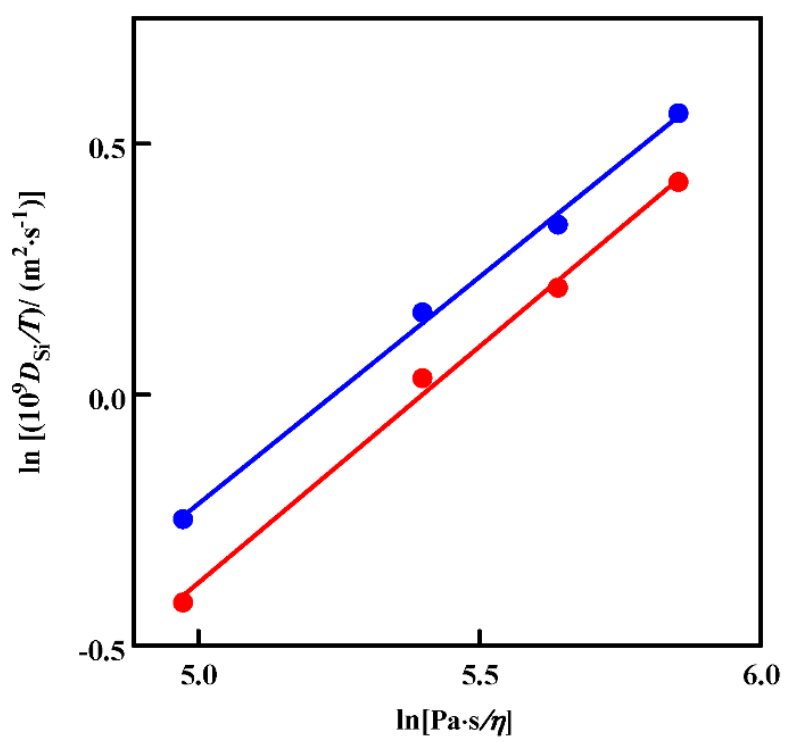

Figure S4 Stokes-Einstein-Sutherland plot of self-diffusion coefficients against fluidity $(1 / \eta)$. The plots for $\mathrm{C}_{1} \mathrm{Im}$ (blue symbols) and $\mathrm{AcOH}$ (red symbols) are linear, showing consistency with the viscosity measurements of Doi et al., ${ }^{1}$ and the slopes are equal within experimental error (0.91 and 0.94 , respectively), showing the consistency between the two data sets. 


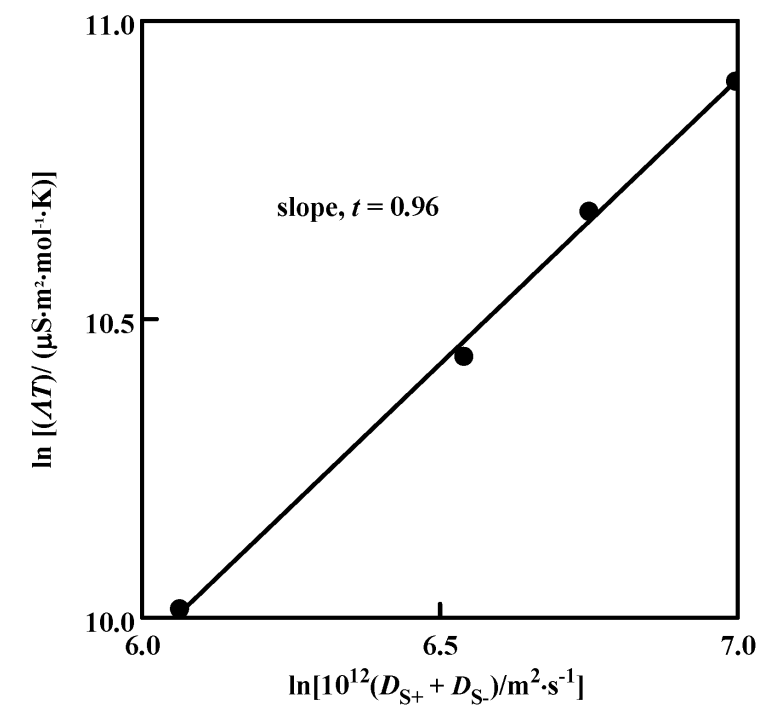

Figure S5 Consistency plot for molar conductivity (Doi et al. ${ }^{1}$ ) and self-diffusion coefficients (this work). Ideally, if the Nernst-Einstein deviation parameter is independent of temperature, the slope is unity. 

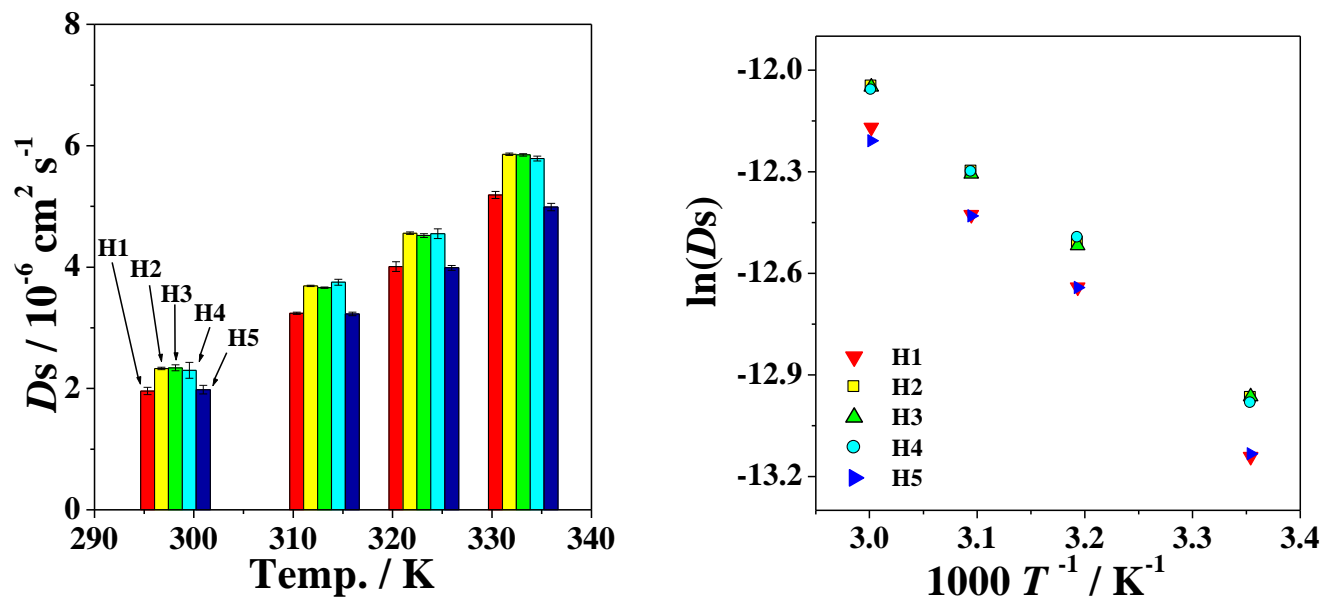

Figure S6. Temperature dependence of the self-diffusion coefficients (a) and their Arrhenius plots (b) for the protons of $\left(\mathrm{C}_{1} \mathrm{Im}+\mathrm{AcOH}\right)$ in the equimolar mixture. 


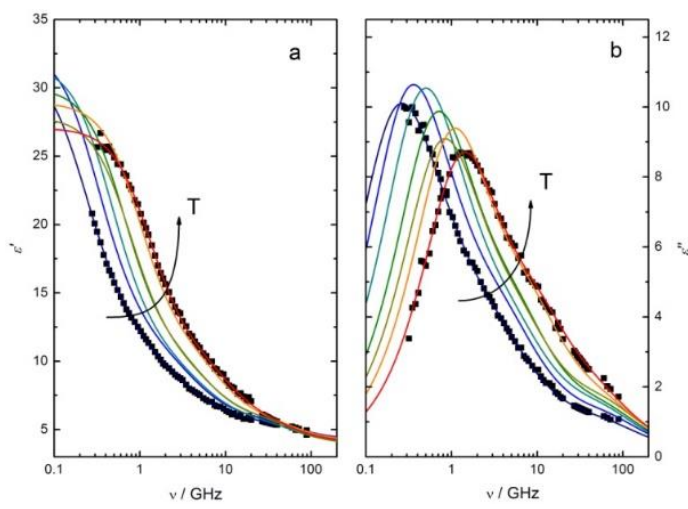

Figure S7 (a) Relative permittivity, $\varepsilon$, and (b) dielectric loss, $\varepsilon$ ", spectra (symbols, partly omitted for clarity) of $\left(\mathrm{C}_{1} \mathrm{Im}+\mathrm{AcOH}\right)$ from 278 to $338 \mathrm{~K}$ in $10 \mathrm{~K}$ steps and their fits (lines) with the CD+D+D model.

\section{Reference :}

(1) Doi, H.; Song, X.; Minofar, B.; Kanzaki, R.; Takamuku, T.; Umebayashi, Y. A New Proton Conductive Liquid with No Ions: Pseudo-Protic Ionic Liquids. Chem. Eur. J. 2013, 19, 11522-11526. (2) Schönert, H. Evaluation of Velocity Correlation Coefficients from Experimental Transport Data in Electrolytic Systems. J. Phys. Chem. 1984, 88, 3359-3363.

(3) Kanakubo, M.; Harris, K. R.; Tsuchihashi, N.; Ibuki, K.; Ueno, M. Effect of Pressure on Transport Properties of the Ionic Liquid 1-Butyl-3- methylimidazolium Hexafluorophosphate. $J$. Phys. Chem. B 2007, 111, 2062-2069.

(4) Harris, K. R.; Kanakubo, M. High Pressure Studies of the Transport Properties of Ionic Liquids. Faraday Discuss. 2012, 154, 425-438. 